\title{
A 1.1 V 81.8 dB Delta-Sigma ADC
}

\author{
Won-Tak Choi and Gil-Cho Ahn \\ Dept. of Electronic Engineering, Sogang University \\ 35 Baekbeom-ro, Mapo-Gu, Seoul 121-742, Korea \\ $\{$ dreamcwt, gcahn\}@sogang.ac.kr
}

\begin{abstract}
A $1.1 \mathrm{~V} 81.8 \mathrm{~dB}$ delta-sigma analog-to-digital converter (ADC) is presented. The split time integration technique for implementing multi-bit digital-to-analog converter (DAC) without using DEM has been developed and used. In order to reduce power consumption and area, a successive approximation register (SAR) ADC is employed to function as both multi-bit quantizer and summing adder without using an additional amplifier. The proposed deltasigma modulator operates at a $640 \mathrm{kHz}$ clock rate and dissipates $850 \mu \mathrm{W}$ with a $1.1 \mathrm{~V}$ supply. It achieves $81.8 \mathrm{~dB}$ dynamic range (DR), $76.8 \mathrm{~dB}$ signal-to-noise and distortion ratio (SNDR) over a $5 \mathrm{kHz}$ signal bandwidth. The core area is $235 \mathrm{\mu m}^{2}$ in a 45-nm CMOS technology.
\end{abstract}

Keywords: delta-sigma ADC, feed-forward, successive approximation register $A D C$

\section{Introduction}

Today's mobile devices are equipped with a lot of embedded sensors, such as a temperature sensor, altimeter, barometer, accelerometer, gyroscope, microphone, and camera which are enabling new features of the devices. In these embedded sensor modules, analog-to-digital converter (ADC) is one of the most important building blocks of the analog front-end (AFE) circuitry. Since the complexity of the AFE is affected by the resolution of the ADC, delta-sigma topology, which provides high dynamic range (DR) by using an oversampling and a noise shaping, is a well suited candidate for sensor interface [1]. Even though the signal bandwidth of delta-sigma modulator is limited by oversampling, it is still wider than the signal bandwidth of sensor output $[2,3]$.

In this paper, a second-order feed-forward delta-sigma modulator with a 4-bit quantizer is proposed. To resolve nonlinearity caused by multi-bit digital-to-analog converter (DAC), a split time integration technique is proposed. A successive approximation register (SAR) type $\mathrm{ADC}$ is used to implement both a 4-bit quantizer and a summing adder without using an additional amplifier.

The paper is organized as follows. Section 2 discusses the architecture of the proposed delta-sigma modulator. In Section 3, the circuit implementation of the modulator is explained. Section 4 describes the measurement results. Finally, conclusions are drawn in Section 5.

\section{Architecture}

Figure 1 shows the proposed second-order delta-sigma modulator. The linearity requirements of the integrators are relaxed by using the feed-forward topology, since the integrators only process the quantization noise [4]. The transfer function of the proposed modulator is given by 


$$
\mathrm{Y}(\mathrm{z})=\mathrm{U}(\mathrm{z})+\left(1-\mathrm{z}^{-1}\right)^{2} \mathrm{Q}(\mathrm{z})
$$

where $\mathrm{Y}(\mathrm{z})$ is an output of the modulator, $\mathrm{U}(\mathrm{z})$ is an input signal and $\mathrm{Q}(\mathrm{z})$ is a quantization noise. In the proposed modulator, a multi-bit quantizer is employed to optimize the performance. However, the nonlinear transfer characteristics of the multi-bit feedback DAC result in performance degradation. To alleviate nonlinear error, dynamic element matching (DEM) technique which randomizes a sequence of mismatch error is commonly used [5-7]. But, the distortion is only converted into random noise and still affects the overall performance [8]. In this paper, split time integration technique using 1-bit DAC is proposed to implement multi-bit DAC without using a DEM. Moreover, a SAR type ADC is used to implement both a 4-bit quantizer and a summing adder without using an additional amplifier.

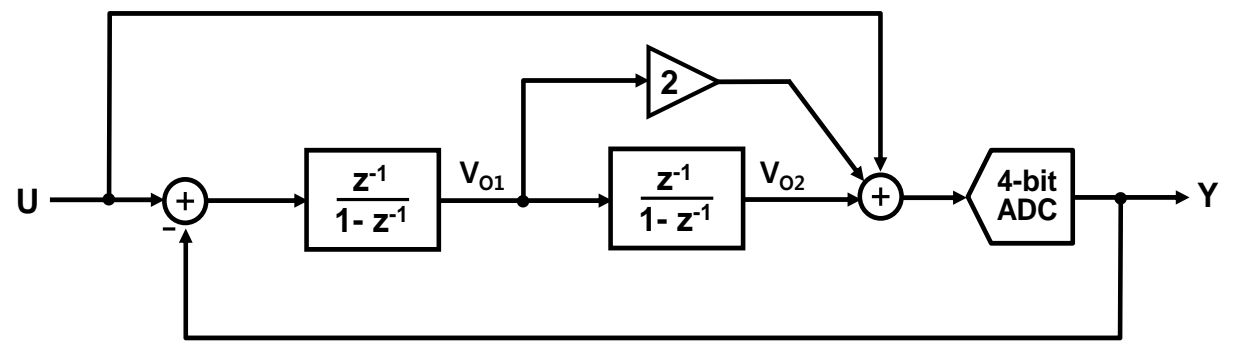

Figure 1. Second-order multi-bit feed-forward DSM

\section{Circuit Implementation}

The proposed delta-sigma modulator which consists of two integrators, a 4-bit SAR ADC is illustrated in Figure 2.

\subsection{Integrator with the proposed multi-bit DAC}

The circuit implementation of the integrator with the proposed multi-bit feedback DAC is shown in Figure 3. The operation is as follows. During the $\phi_{1}$ phase, input signal is sampled into the sampling capacitors $\mathrm{C}_{S}$ while the 8-bit control signal for DAC operation is generated from 4-bit output of the quantizer.During the next $\phi_{2}$ phase, integrating DAC operates with $\phi_{3}$ and $\phi_{4}$ clocks, while sampled input signal is transferred to feedback capacitor $\mathrm{C}_{\mathrm{F}}$ with $\phi_{2}$ clock. During the $\phi_{3}$ phase, reference voltage is sampled into DAC capacitor $C_{D}$ according to 1-bit control signal, $D_{k}$, and sampled charge in $C_{D}$ is integrated into $C_{F}$ during the $\phi_{4}$ phase. These 1-bit DAC operation repeats 8 times during the $\phi_{2}$ phase. The output of the proposed integrator with an ideal opamp is given by

$$
\mathrm{V}_{\text {OUT }}(\mathrm{n})=\mathrm{V}_{\text {OUT }}(\mathrm{n}-1)+\frac{\mathrm{C}_{\mathrm{S}}+\Delta \mathrm{C}_{\mathrm{S}}}{\mathrm{C}_{\mathrm{F}}} \mathrm{V}_{\mathrm{IN}}(\mathrm{n}-1)+\frac{\mathrm{C}_{\mathrm{D}}+\Delta \mathrm{C}_{\mathrm{D}}}{\mathrm{C}_{\mathrm{F}}} \sum_{\mathrm{k}=0}^{7} \mathrm{D}_{\mathrm{k}} \mathrm{V}_{\mathrm{REF}}
$$

where $\Delta \mathrm{C}_{\mathrm{D}}$ is the mismatch error of DAC capacitor, $\Delta \mathrm{C}_{\mathrm{S}}$ is the mismatch error of the sampling capacitor. The gain of the proposed split time integration DAC can be found from (2) as

$$
\mathrm{G}_{\mathrm{DAC}}=\frac{\mathrm{C}_{\mathrm{D}}+\Delta \mathrm{C}_{\mathrm{D}}}{\mathrm{C}_{\mathrm{F}}}
$$


As shown in (3), it provides a constant gain error for DAC operation which has a linear transfer characteristic [9].

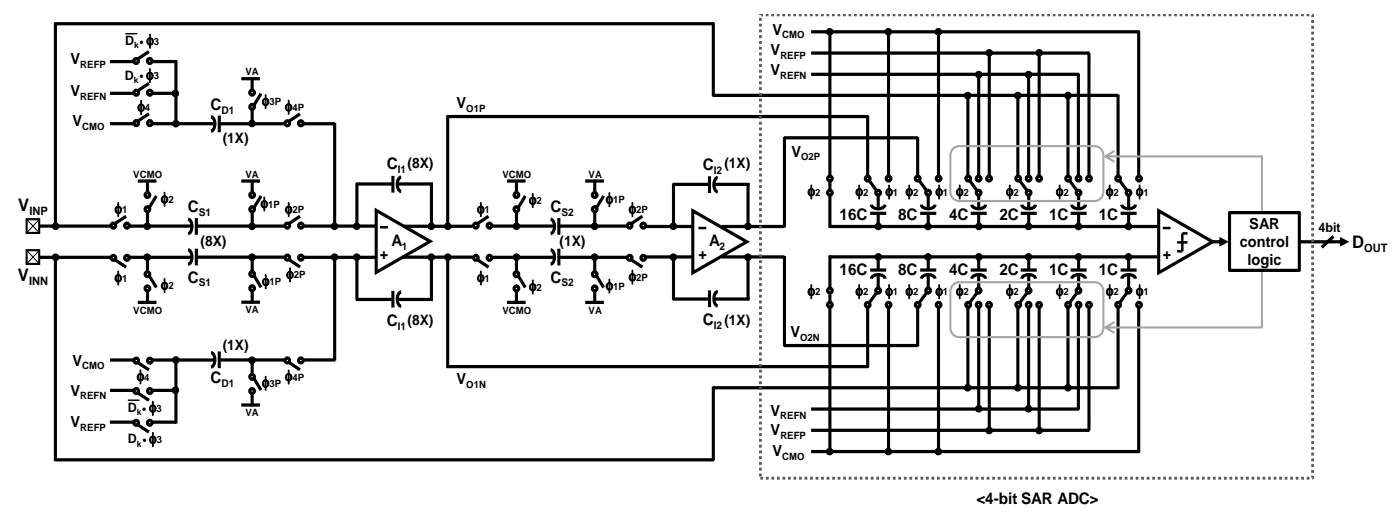

Figure 2. Circuit implementation of the proposed delta-sigma modulator
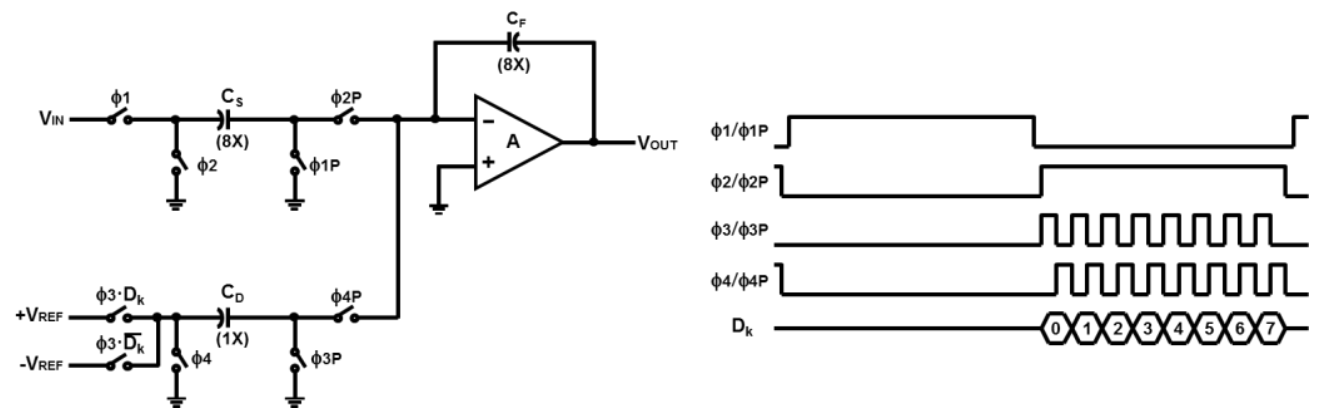

Figure 3. Circuit implementation of the integrator with the proposed multi-bit DAC

\subsection{Proposed multi-bit quantizer using SAR ADC}

The proposed 4-bit SAR ADC which includes a summing adder is shown in Figure 2. It consists of a binary weighted capacitor array, a comparator and SAR control logic. The operation of the proposed SAR ADC is as follows. During the $\phi_{2}$ phase, the top plates are reset to the common-mode voltage, $\mathrm{V}_{\mathrm{CMO}}$, while the bottom plates are connected to each signal. During the next $\phi_{1}$ phase, the top plates are disconnected from $\mathrm{V}_{\mathrm{CMO}}$ and the bottom plates of the capacitors are switched to $\mathrm{V}_{\mathrm{CMO}}$ and then comparator decides most significant bit (MSB). After the decision of MSB, bottom plates of $4 \mathrm{C}, 2 \mathrm{C}$ and $\mathrm{C}$ are switched to $\mathrm{V}_{\mathrm{REFP}}$ or $\mathrm{V}_{\text {REFN }}$ according to the previous comparator output [10]. The comparator input signal after the completion of the conversion is given

$$
\mathrm{V}_{+}-\mathrm{V}_{-}=\frac{1}{4}\left[\left(\mathrm{~V}_{\mathrm{IN}}+2 \mathrm{~V}_{\mathrm{O} 1}+\mathrm{V}_{\mathrm{O} 2}\right)-\left(\frac{1}{2} \mathrm{D}_{3}+\frac{1}{4} \mathrm{D}_{2}+\frac{1}{8} \mathrm{D}_{1}\right) \cdot \mathrm{V}_{\mathrm{REF}}\right]
$$

where, $\mathrm{V}_{\mathrm{IN}}=\mathrm{V}_{\mathrm{INP}}-\mathrm{V}_{\mathrm{INN}}, \mathrm{V}_{\mathrm{O} 1}=\mathrm{V}_{\mathrm{O} 1 \mathrm{P}}-\mathrm{V}_{\mathrm{O} 1 \mathrm{~N}}$ and $\mathrm{V}_{\mathrm{O} 2}=\mathrm{V}_{\mathrm{O} 2 \mathrm{P}}-\mathrm{V}_{\mathrm{O} 2 \mathrm{~N}}$. A timing generation for the proposed SAR ADC is facilitated by using an asynchronous control using internal loop delay. 


\section{Measurement Results}

The prototype delta-sigma modulator was fabricated in a 45-nm CMOS technology. Figure 4 shows a measured output spectrum for a $1 \mathrm{kHz} 640 \mathrm{mV}$ peak-to-peak differential input sine wave, with a 1.1-V power supply. The SNR and SNDR versus input level curve is illustrated in Figure 5. This prototype IC achieves $76.8 \mathrm{~dB}$ of SNDR, $82.2 \mathrm{~dB}$ of SFDR and $81.8 \mathrm{~dB}$ of DR. Figure 6 shows the layout of the proposed ADC. The core area occupies $235 \mu \mathrm{m}^{2}$. The total power consumption is $850 \mu \mathrm{W}$. The overall measured results are summarized in Table 1 .

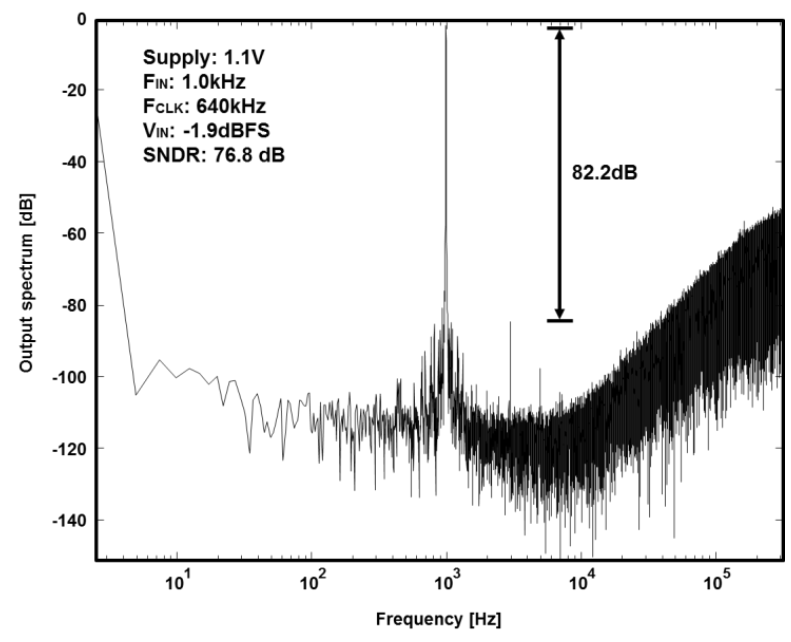

Figure 4. Measured output spectrum

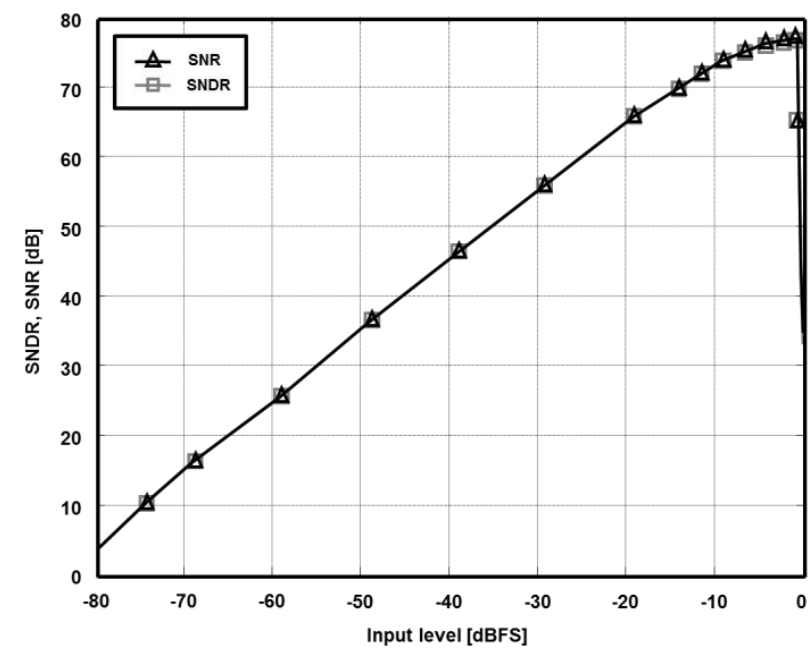

Figure 5. SNR and SNDR versus input level 


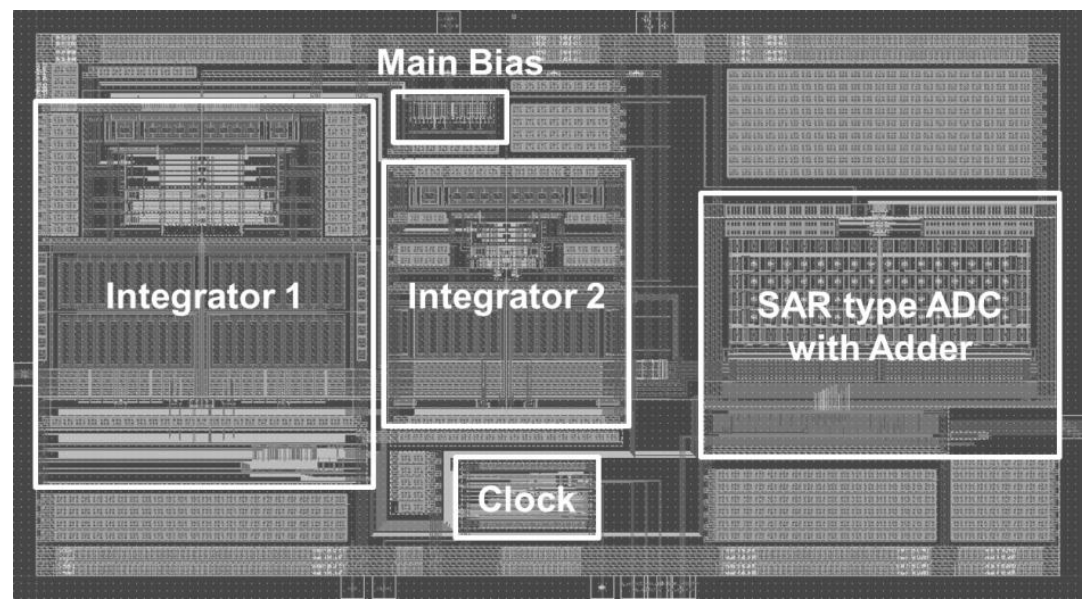

Figure 6. Chip layout

Table 1. Performance summary

\begin{tabular}{c|c||c|c}
\hline Process & 45-nm CMOS & Peak SNR & $77.8 \mathrm{~dB}$ \\
\hline Power supply & $1.1 \mathrm{~V}$ & Peak SNDR & $76.8 \mathrm{~dB}$ \\
\hline Clock & $640 \mathrm{kHz}$ & Peak SFDR & $82.2 \mathrm{~dB}$ \\
\hline Signal bandwidth & $5 \mathrm{kHz}$ & $\begin{array}{c}\text { Power } \\
\text { consumption }\end{array}$ & $\begin{array}{c}590 \mu \mathrm{W} \text { (Analog) } \\
260 \mu \mathrm{W} \text { (Digital) }\end{array}$ \\
\hline OSR & 64 & Chip Area & $235 \mu \mathrm{m}^{2}$ \\
\hline DR & $81.8 \mathrm{~dB}$ &
\end{tabular}

\section{Conclusion}

A $1.1 \mathrm{~V} 81.8 \mathrm{~dB}$ delta-sigma modulator is presented in this paper. The split time integration technique is adopted to implement a multi-bit DAC without using a DEM. In order to minimize power consumption and area, a 4-bit asynchronous SAR ADC is used for quantizer with summing operation. The measurement results of the prototype ADC fabricated in a 45-nm CMOS technology verify the validity of the proposed design technique.

\section{Acknowledgements}

This work was supported by the Samsung Electronics Co. and MSIP (Ministry of Science, ICT \& Future Planning), Korea, under the University ITRC support program (NIPA-2013H0301-13-1007) supervised by the NIPA (Nation IT Industry Promotion Agency).

\section{References}

[1] R. Schreier and G. C. Temes, "Understanding Delta-sigma Data Converters", IEEE Press/Wiley Interscience, (2005).

[2] Y. Kwak and G. Ahn, "A 1.8V 89.2 dB Delta-Sigma ADC for Sensor Interface with On-Chip Reference", IEEE Int. Symp. Circuits Syst., vol. 1, (2012) May 20-23, pp. 520-523.

[3] J. M. de la Rosa, S. Escalera, B. Perez-Verdu, F. Medeiro, O. Guerra, R. del Rio and A. Rodriguez-Vazquez, "A CMOS 110-dB@40-kS/s Programmable-Gain Chopper-Stabilized Third-Order 2-1 Cascade Sigma-Delta Modulator for Low-Power High-Linearity Automotive Sensor ASICs”, IEEE J. Solid-State Circuits, vol. 40, no. 11, (2005) November, pp. 2246-2264.

[4] J. Silva, U. Moon, J. Steensgaard and G. Temes, "Wideband low-distortion delta-sigma ADC topology", Electron.Lett., vol. 37, no. 12, (2001) June, pp. 737-738. 
[5] A. J. Chen and Y. P. Xu, "Multibit delta-sigma modulator with noise-shaping dynamic element matching", IEEE Trans. Circuits Syst. I, Reg. Papers, vol. 56, no. 6, (2009) June, pp. 1125-1133.

[6] S. Yoo, G. Noh, K. Kim and G. Ahn, "A 1.6V $\Delta \Sigma$ ADC for Digital Electret Microphone”, International SoC Design Conf. (ISOCC), (2010) November, pp. 283-286.

[7] R. J. Van De Plassche, "Dynamic element matching for high accuracy monotonic D/A converters", IEEE J. Solid-State Circuits, vol. 11, no. 6, (1976) June, pp. 795-800.

[8] I. Galton, "Digital cancellation of D/A converter noise in pipelined A/D converters", IEEE Trans. Circuits Syst. II, vol. 47, (2000) March, pp. 185-196.

[9] J. McCreary and P. Gray, "All-MOS charge redistribution analog-to-digital conversion techniques", IEEE J. Solid-State Circuits, vol. SC-10, no. 12, (1975) December, pp. 371-379.

[10] W. Choi, E. Choe and G. Ahn, "A Low-Distortion Second-Order Multi-Bit Delta-Sigma ADC", Advanced Science and Technology Letters, vol. 43, (2013) December, pp. 63-66.

[11] J. McCreary and P. Gray, "All-MOS charge redistribution analog-to-digital conversion techniques", IEEE J. Solid-State Circuits, vol. SC-10, no. 12, (1975) December, pp. 371-379.

\section{Authors}

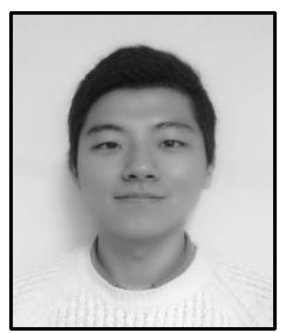

\section{Won-Tak Choi}

He received the B.S. degree in the Department of Electrical Engineering from Sogang University, Seoul, Korea, in 2012. He is currently pursuing the M.S. degree in Electronic Engineering from Sogang University, Korea. His research interests include CMOS integrated circuit for audio applications and analog mixed-signal ICs.

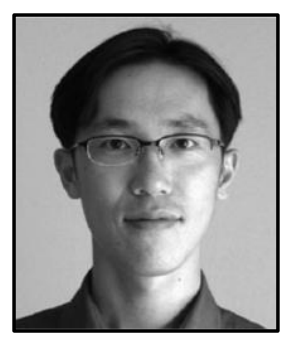

\section{Gil-Cho Ahn}

He received the B.S. and M.S. degrees in electronic engineering from Sogang University, Seoul, Korea, in 1994 and 1996, respectively, and the Ph.D. degree in electrical engineering from Oregon State University, Corvallis, in 2005.From 1996 to 2001, he was a Design Engineer at Samsung Electronics, Kiheung, Korea, working on mixed analog-digital integrated circuits. From 2005 to 2007, he was with Broadcom Corporation, Irvine, CA, working on AFE for digital TV. Currently, he is an Assistant Professor in the Department of Electronic Engineering, Sogang University. His research interests include high-speed, highresolution data converters and low-voltage, low-power mixed-signal circuits design. 